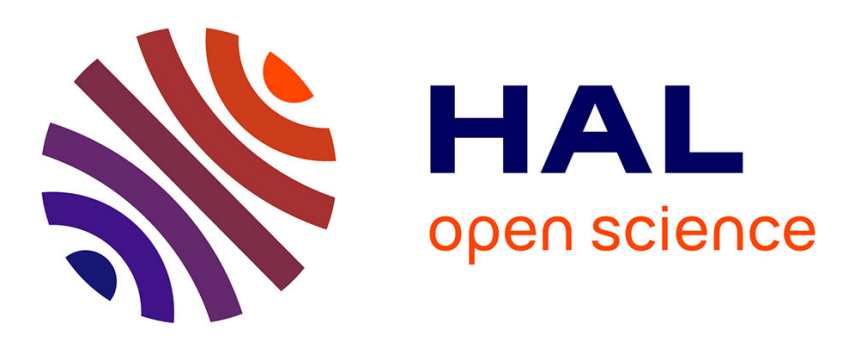

\title{
Diffraction radiation from ultrarelativistic particles passing through a slit. Determination of the electron beam divergence
}

\author{
N. Potylitsina-Kube, X. Artru
}

\section{- To cite this version:}

N. Potylitsina-Kube, X. Artru. Diffraction radiation from ultrarelativistic particles passing through a slit. Determination of the electron beam divergence. Nuclear Instruments and Methods in Physics Research Section B: Beam Interactions with Materials and Atoms, 2003, 201, pp.172-183. 10.1016/S0168-583X(02)01519-7 . in2p3-00012304

\section{HAL Id: in2p3-00012304 https://hal.in2p3.fr/in2p3-00012304}

Submitted on 25 Feb 2003

HAL is a multi-disciplinary open access archive for the deposit and dissemination of scientific research documents, whether they are published or not. The documents may come from teaching and research institutions in France or abroad, or from public or private research centers.
L'archive ouverte pluridisciplinaire HAL, est destinée au dépôt et à la diffusion de documents scientifiques de niveau recherche, publiés ou non, émanant des établissements d'enseignement et de recherche français ou étrangers, des laboratoires publics ou privés. 


\title{
Diffraction Radiation from ultrarelativistic particles passing through a slit. Determination of the electron beam divergence.
}

\author{
N. Potylitsina-Kube, ${ }^{1}$ X. Artru \\ Tomsk Polytechnic University, 634004, Lenin ave. 30, Tomsk, Russia \\ Institut de Physique Nucléaire de Lyon, \\ 4 rue Enrico Fermi, 69622 Villeurbanne Cedex, France
}

\begin{abstract}
In the present article the available formulas for diffraction radiation (DR) by an electron near a perfectly conducting half plane are generalized to any direction of the electron velocity, using Lorentz transformation. This allows to take into account electron beam divergence in an exact way. A new method for determining both horizontal and vertical beam divergence using one slit DR radiator, but two optical wavelengths, is proposed.
\end{abstract}

Key words: Diffraction radiation, Beam divergence, Emittance PACS: $51.60 \mathrm{Nr}$

\section{Introduction}

The problem of precision diagnostics of low emittance electron beams is rather pressing as it is planned in the nearest future to produce electron- positron linear colliders and X-ray free electron lasers using electron beams of the record parameters.

Nowadays the beam diagnostics based on optical transition radiation (OTR) has been used rather widely [1]-[3]. This is due to the fact that OTR has such advantages as : (i) a well developed theory allowing to obtain information on electron beam parameters using the characteristics of optical transition radiation (ii) comparatively simple equipment and (iii) an opportunity to carry out measurements in low background conditions using so called backward transition radiation emitted at a large angle with respect to the electron beam. But the use of a solid target for OTR generation (such as foil) inevitably results in impairing the beam parameters.

During the last years a new method of non-invasive beam diagnostics has been developed. The

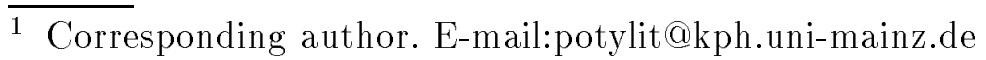


method is based on the diffraction radiation (DR) of ultrarelativistic electrons, i.e. radiation appearing when a charged particle is moving close to the edge of a screen. Up to now the theory has been developed for the case of a perfectly conducting target (semi-infinite screen or slit in infinite screen, see [4]-[11] for example).

The angular distribution of optical DR has been calculated by M. Castellano for an electron beam passing through a slit in the screen which is oriented perpendicular to the beam axis, assuming a Gaussian beam profile in the transverse directions [7]. The point of investigation in this work was the angular distribution of DR which is emitted close to the direction of the initial electron beam. The application of that geometry to beam diagnostic measurements necessitates either to deflect the downstream electron beam, resulting in an additional background due to the emission of synchrotron radiation, or to deflect the DR light with a mirror close to the beam, producing additional DR from the mirror edge.

In the case of OTR beam diagnostics, the simplest way to avoid these additional radiations is to use the backward radiation geometry, in which the target (i.e. a foil) is tilted, typically at an angle $\varphi_{0}=45^{\circ}$ from the beam axis, and optical transition radiation is detected at an angle $\varphi_{D}=2 \cdot \varphi_{0}=90^{\circ}$. As it is shown in Ref.[8] by a complete analogy with transition radiation, the DR angular distribution of ultrarelativistic particles has the form of two cones with a characteristic apex angle $\sim \gamma^{-1}, \gamma$ is the Lorentz factor, one of them being centered along the direction of the initial particle velocity (forward DR), and the other one along the direction of specular reflection from the target plane (backward DR). It is obvious from the discussion above that the preferable geometry for beam diagnostics measurements is that one investigating the backward DR component.

In Ref.[9] the authors investigated the backward DR angular distribution for a slit under the influence of the electron beam divergence, assuming the divergence takes place in the plane perpendicular to the slit orientation. In Ref.[10] R.B.Fiorito and D.W.Rule proposed a method for measuring the electron beam divergences and sizes in the two transverse directions, making use of two DR slit radiators and analyzing the polarization of the radiation. These authors present a simple approximate method of calculation of backward DR. In Ref.[12] R.B.Fiorito also considered the interference between forward DR of a first radiator and backward transition radiation of a second radiator as a promising tool for beam diagnostics.

Exact formulas for the DR spectral-angular distribution of a particle moving close to a semiinfinite ideally conducting target were obtained more than 30 years ago [4], [5]. The obtained formulas represent two special cases. The first one considers a geometry in which the projection of the electron trajectory onto the target plane is perpendicular to the target edge (perpendicular case, $\psi_{0}=\frac{\pi}{2}, \varphi_{0}$ arbitrary; Fig.1a). In the second case the projection is parallel to the target edge (parallel case, $\psi_{0}$ arbitrary $; \varphi_{0}=\frac{\pi}{2}$; Fig. $1 \mathrm{~b}$ ). It is evident that for precision diagnostics purposes it is necessary to obtain general formulas describing the DR characteristics for any incoming angles of electrons. It will allow to analyze the influence of a real electron beam with finite divergences in the two perpendicular planes.

Later in Ref.[8] it was shown that in the ultrarelativistic approximation $(\gamma \gg 1)$ the formulas of DR spectral-angular distribution for both geometries coincide. The authors of [10] also reproduce this formula and use it for geometries which differ from the two above-mentioned special ones for the case when an electron beam has both horizontal and vertical divergences . In the present article, using Lorentz transformation, we will get the generalization of the exact formula [4] to any geometry, i.e., we will give the DR spectral-angular distribution when the projection of an electron trajectory onto the target plane is inclined at any angle with respect to the target edge. This will allow to calculate the effect of the initial electron beam 
divergence on the DR radiation characteristics for any electron energy, and in the case $\gamma \gg 1$, to justify the use of an universal approximate formula for DR.

\section{DR spectral-angular distribution, general case.}

Let us consider the perpendicular case (Fig.1a), where $\varphi_{0}$ is the azimuthal angle between the electron beam and the target plane. As it is shown in [4], in this case the DR spectral-angular distribution is described by the following expression:

$$
\begin{gathered}
\frac{d^{2} W}{d \omega d \Omega}=\frac{\alpha}{4 \pi^{2}} \frac{\beta}{\sin \psi} \exp \left(-\frac{4 \pi h}{\lambda \beta} \sqrt{1-\beta^{2} \sin ^{2} \psi}\right) \times \\
\times \frac{\left(1-\beta^{2} \sin ^{2} \psi\right)\left(1+\beta_{x} \sin \psi\right)(1-\cos \varphi)+\cos ^{2} \psi\left(1-\beta_{x} \sin \psi\right)(1+\cos \varphi)}{\left(1-\beta^{2} \sin ^{2} \psi\right)\left[\left(1-\beta_{x} \sin \psi \cos \varphi\right)^{2}-\beta_{y}^{2} \sin ^{2} \psi \sin ^{2} \varphi\right]}
\end{gathered}
$$

The system of units used in Eq.(1) and in the following is $\hbar=c=1 ; \vec{\beta}=\left\{\beta_{x}, \beta_{y}, 0\right\}=$ $\beta\left\{\cos \varphi_{0}, \sin \varphi_{0}, 0\right\}, \beta$ is the electron velocity, $\varphi, \psi$ are the azimuthal and polar angles of photon emission as shown in Fig. 2 ; $\omega$ is the DR photon energy; $\gamma$ the Lorentz factor; $\alpha$ the fine structure constant; $h$ the shortest distance to the target. The dependence on the electron angle $\varphi_{0}$ with respect to the target plane is expressed in Eq.(1) by the components $\beta_{x}, \beta_{y}$. Let us now consider the case when the electron moves strictly perpendicular to the target plane $\vec{\beta}=\left\{0, \beta_{y}, 0\right\}$. Then, in the system of coordinates of an observer which moves along the $Z$-axis with velocity $\vec{V}$, according to the Lorentz transformation (LT), the electron velocity will have two components $\left(0, \beta_{y}^{\prime}=\beta_{y} \sqrt{1-\beta_{z}^{\prime 2}}, \beta_{z}^{\prime}=\frac{V}{c}\right)$. Thus, in a moving system, where all variables are indicated by a prime, we have the parallel case considered in Ref.[5]. The validity of such an approach rely on the homogenity of the screen along the $Z$-axis and the invariance of the boundary conditions for the total field at the surface of the screen,

$$
E_{x}=E_{z}=B_{y}=0
$$

under the considered LT (strictly speaking, this invariance needs a perfectly conducting screen). Consequently, in any reference frame moving along that axis the Maxwell equations will describe the same processes as in the laboratory frame. So, using the Lorentz transformations:

$$
\begin{gathered}
\sin \psi=\frac{\sqrt{1-\beta_{z}^{\prime 2}} \sin \psi^{\prime}}{1-\beta_{z}^{\prime} \cos \psi^{\prime}}, \cos \psi=\frac{\cos \psi^{\prime}-\beta_{z}^{\prime}}{1-\beta_{z}^{\prime} \cos \psi^{\prime}} \\
\omega=\frac{\omega^{\prime}\left(1-\beta_{z}^{\prime} \cos \psi^{\prime}\right)}{\sqrt{1-\beta_{z}^{\prime 2}}}, \beta_{x, y}=\frac{\beta_{x, y}^{\prime}}{\sqrt{1-\beta_{z}^{\prime 2}}}
\end{gathered}
$$

and changing the unprimed components in Eq.(1) with the primed ones according to formulas (2) we get:

$$
\left.\frac{d^{2} W}{d \Omega d \omega}\right|_{\left(0, \beta_{y}, 0\right)} ^{(\perp \text { case })} \stackrel{L T}{\rightarrow} \frac{\alpha}{4 \pi^{2}} \frac{\beta_{y}^{\prime}\left(1-\beta_{z}^{\prime} \cos \psi^{\prime}\right)}{\sin \psi^{\prime}} \exp \left(-\frac{4 \pi h}{\lambda^{\prime} \beta_{y}^{\prime}} \sqrt{\left(1-\beta_{z}^{\prime} \cos \psi^{\prime}\right)^{2}-\beta_{y}^{\prime 2} \sin ^{2} \psi}\right) \times
$$




$$
\begin{gathered}
\times \frac{\left[\left(1-\beta_{z}^{\prime} \cos \psi^{\prime}\right)^{2}-\beta_{y}^{\prime 2} \sin ^{2} \psi^{\prime}\right](1-\cos \varphi)+\left(\cos \psi^{\prime}-\beta_{z}^{\prime}\right)^{2}(1+\cos \varphi)}{\left[\left(1-\beta_{z}^{\prime} \cos \psi^{\prime}\right)^{2}-\beta_{y}^{\prime 2} \sin ^{2} \psi^{\prime}\right]\left[\left(1-\beta_{z}^{\prime} \cos \psi^{\prime}\right)^{2}-\beta_{y}^{\prime 2} \sin ^{2} \psi^{\prime} \sin ^{2} \varphi\right]} \cdot \frac{\overbrace{\left(1-\beta_{z}^{\prime} \cos \psi^{\prime}\right)^{2}}^{\omega^{2}}}{1-\beta_{z}^{\prime 2}} \cdot \frac{1}{\omega^{\prime 2}}= \\
=\left.\frac{d^{2} W}{d \Omega^{\prime} d \omega^{\prime}}\right|_{\left(0, \beta_{y}^{\prime}, \beta_{z}^{\prime}\right)} ^{(\text {non } \perp \text { case })} \cdot \frac{\omega^{2}}{\omega \prime^{2}}
\end{gathered}
$$

The last sign of equality follows from the known Lorentz invariance of $\frac{d^{2} W}{d \Omega d \omega} \cdot \frac{1}{\omega^{2}}$. Comparing the obtained expression with the results [5] for the parallel case we see a complete identity of the formulas for the DR spectral density. Performing the same consideration for the perpendicular case, but this time with two nonzero components of velocity $\vec{\beta}=\left(\beta_{x}, \beta_{y}, 0\right)$, we obtain the general case, i.e. the velocity projection has an arbitrary angle with respect to the target edge (in other words the electron velocity has three nonzero projections). The spectral-angular density for the general case (Fig.2), i.e. for $\vec{\beta}=\left\{\beta_{x}, \beta_{y}, \beta_{z}\right\}=\beta\left\{\cos \varphi_{0} \sin \psi_{0}, \sin \varphi_{0} \sin \psi_{0}, \cos \psi_{0}\right\}$ is expressed as:

$$
\begin{gathered}
\frac{d^{2} W}{d \omega d \Omega}=\frac{\alpha}{4 \pi^{2}} \frac{\beta_{\perp}\left(1-\beta_{z} \cos \psi\right)}{\sin \psi} \exp \left(-\frac{4 \pi h}{\lambda \beta_{\perp}} \sqrt{\left(1-\beta_{z} \cos \psi\right)^{2}-\beta_{\perp}^{2} \sin ^{2} \psi}\right) \\
\times \frac{\left[\left(1-\beta_{z} \cos \psi\right)^{2}-\beta_{\perp}^{2} \sin ^{2} \psi\right]\left(1+\frac{\beta_{x} \sin \psi}{1-\beta_{z} \cos \psi}\right)(1-\cos \varphi)+\left(\cos \psi-\beta_{z}\right)^{2}\left(1-\frac{\beta_{x} \sin \psi}{1-\beta_{z} \cos \psi}\right)(1+\cos \varphi)}{\left[\left(1-\beta_{z} \cos \psi\right)^{2}-\beta_{\perp}^{2} \sin ^{2} \psi\right]\left[\left(1-\beta_{z} \cos \psi-\beta_{x} \sin \psi \cos \varphi\right)^{2}-\beta_{y}^{2} \sin ^{2} \psi \sin ^{2} \varphi\right]}
\end{gathered}
$$

Here $\beta_{\perp}^{2}=\beta_{x}^{2}+\beta_{y}^{2}$. It is obvious that Eq.(4) includes both geometries as special cases: the perpendicular one for $\beta_{z}=0$ and the parallel one for $\beta_{x}=0$. Note that the spectral-angular distribution is even in $\varphi$, implying the symmetry between forward and backward DR with respect to the screen plane (for a perfect infinite thin screen).

\section{Ultrarelativistic approximation}

The spectral-angular distribution of Eq.(4) attains its maximum value near the place where the denominator is minimum. As pointed out in Ref.[8], this gives two symmetrical cones of maximal intensity, centered at $\psi=\psi_{0}, \varphi= \pm \varphi_{0}$. Accordingly, for "forward" DR, we write

$$
\varphi=\varphi_{0}+\frac{\theta_{\varphi}}{\sin \psi_{0}}, \quad \psi=\psi_{0}+\theta_{\psi}
$$

whereas for radiation along the specular direction ("backward" DR):

$$
\varphi=-\varphi_{0}+\frac{\theta_{\varphi}}{\sin \psi_{0}}, \quad \psi=\psi_{0}+\theta_{\psi}
$$

with $\theta_{\psi}, \theta_{\varphi} \ll 1$, as shown in Fig.2. As an example let us consider "backward" radiation. In the ultrarelativistic approximation $\gamma \gg 1$ the variables $\theta_{\psi}, \theta_{\varphi}$ are of the order $\sim \gamma^{-1}$. 
Expansion of numerator and denominator in Eq.(4) up to the order of $\gamma^{-2}$ results in the following expression:

$$
\left.\frac{d^{2} W}{d \omega d \Omega}\right|_{\text {one edge }}=\frac{\alpha}{4 \pi^{2}} \exp \left(-\frac{\omega}{\omega_{c}} \sqrt{1+\gamma^{2} \theta_{\psi}^{2}}\right) \frac{\gamma^{-2}+2 \theta_{\psi}^{2}}{\left(\gamma^{-2}+\theta_{\psi}^{2}\right)\left(\gamma^{-2}+\theta_{\psi}^{2}+\theta_{\varphi}^{2}\right)}
$$

Here $\omega_{c}=\frac{\gamma}{2 h}$ is the characteristic energy of the DR spectrum. It should be noted that in Eq.(7) the angles $\theta_{\psi}, \theta_{\varphi}$ are counted from the specular direction. It is important to notice that in the ultrarelativistic approximation the DR spectral intensity from a semi-infinite half plane for the general case, Eq.(7), is the same than for the perpendicular and the parallel geometry, cf. Ref.[8]. As a consequence, considering DR from a slit target for the general case it is sufficient to adopt the results derived in Ref.[9] for the perpendicular case, as we will point out in the next chapter.

\section{DR from a slit}

Let us now consider the case when the electron beam passes through a slit in a tilted target in the geometry shown in Fig.3a. The angles between one individual electron trajectory and the target plane are

$$
\psi_{0}=\bar{\psi}_{0}+\Delta_{\psi}, \quad \varphi_{0}=\bar{\varphi}_{0}+\Delta_{\varphi}
$$

with the beam divergences $\Delta_{\psi}, \Delta_{\varphi} \ll 1$ and the coordinates of the beam axis $\bar{\psi}_{0}, \bar{\varphi}_{0}$. As shown in the previous section the DR intensity for the semi-infinite half plane in the ultrarelativistic approximation does not depend on the geometry. Therefore, in the case shown in Fig.3a the single electron spectral-angular distribution from the slit is given by the same expression than for the exact perpendicular geometry, $\left(\psi_{0}=\frac{\pi}{2}\right)$, from Ref.[10]. The only modification is that for the general case the angular deviations $\theta_{\psi}, \theta_{\varphi}$ are defined as follows:

$$
\theta_{\psi}=\psi-\psi_{0} \quad, \quad \theta_{\varphi}=\left(\varphi+\varphi_{0}\right) \sin \psi_{0},
$$

while for the target with a slit considered in Ref.[10] (perpendicular geometry) the introduced angles are defined as $\theta_{\psi}=\psi-\frac{\pi}{2}, \theta_{\varphi}=\varphi+\varphi_{0}$.

The DR spectral-angular density from the slit in the general case is therefore expressed as

$$
\begin{gathered}
\left.\frac{d^{2} W}{d \omega d \Omega}\right|_{s l i t}=\frac{\alpha \gamma^{2}}{4 \pi^{2}} \frac{\exp \left(-\frac{\omega}{\omega_{c}} \sqrt{1+t_{\psi}^{2}}\right)}{\left(1+t_{\psi}^{2}\right)\left(1+t_{\psi}^{2}+t_{\varphi}^{2}\right)} \times \\
\times\left\{2\left(1+2 t_{\psi}^{2}\right) \cosh \left(2 l \frac{\omega}{\omega_{c}} \sqrt{1+t_{\psi}^{2}}\right)-\frac{2}{\left(1+t_{\psi}^{2}+t_{\varphi}^{2}\right)}\left[\left(1+t_{\psi}^{2}-t_{\varphi}^{2}\right) \cos \left(\frac{\omega}{\omega_{c}} t_{\varphi}\right)-2 t_{\varphi} \sqrt{1+t_{\psi}^{2}} \sin \left(\frac{\omega}{\omega_{c}} t_{\varphi}\right)\right]\right\} \\
=I\left(K, l, t_{\psi}, t_{\varphi}\right) .
\end{gathered}
$$

Here $K$ stands for a set of parameters $\left(\omega, \omega_{c}, \gamma\right), t_{\psi, \varphi}=\gamma \cdot \theta_{\psi, \varphi}$ and $l$ is a relative parameter which characterizes the particle coordinates with respect to the slit center, $l=\frac{h_{1}}{a \sin \varphi_{0}}-\frac{1}{2}$, $(-0.5<l<0.5)$. The characteristic DR energy is expressed as $\omega_{c}=\frac{\gamma}{a \sin \varphi_{0}}$ and the slit width 
as $a=\frac{h_{1}+h_{2}}{\sin \varphi_{0}}$, where $h_{1,2}$ is the minimum distance to the upper (lower) half plane (Fig.3a). In the limit of an infinitely narrow slit, Eq.(8) reduces to the formula for transition radiation of a perfectly conducting mirror,

$$
\frac{d^{2} W}{d \omega d \Omega} \simeq \frac{\alpha}{\pi^{2}}\left(\frac{\theta}{\gamma^{-2}+\theta^{2}}\right)^{2} \quad(\gamma \gg 1, \theta \ll 1)
$$

with $\theta^{2}=\theta_{\psi}^{2}+\theta_{\varphi}^{2}$. Formula (8) agrees with results of Ref.[10], which are written for small $l$. It is valid for $\theta \ll 1, \gamma \gg 1$ and for any orientation of the electron beam with respect to the slit. In this case one should use the angles $\psi_{0}=\bar{\psi}_{0}+\Delta_{\psi}, \varphi_{0}=\bar{\varphi}_{0}+\frac{\Delta_{\varphi}}{\sin \psi_{0}}$ in Figs.6,7. It follows from Eq.(8) that the characteristics of DR explicitly depends on the distance between the electron trajectory and the center of the slit, described by the impact parameter $h_{0}=l \cdot a \cdot \sin \varphi_{0}(\mathrm{cf}$. Fig.3b). In the next section, Eq.(8) is used to simulate the DR characteristics and to study the influence of the electron beam divergence and size.

\section{Simulation of the DR characteristics}

Let us consider the influence of the initial electron beam divergence and size. If the electron beam has zero divergence and is characterized by the impact parameter distribution $G\left(h_{0}\right)$ (Fig.3b), then the spectral-angular density of the emitted DR beam is determined by folding expression (9) with $G\left(h_{0}\right)$ :

$$
\widehat{I}\left(K, t_{\psi}, t_{\varphi}\right)=\int I\left(K, h_{0}, t_{\psi}, t_{\varphi}\right) G\left(h_{0}\right) d h_{0} .
$$

The integration extends over the beam size. We describe the divergent electron beam using a Gaussian distribution centered around the averaged direction (Fig.4)

$$
<\vec{\beta}>=<\beta>\left\{\sin \bar{\psi}_{0} \cos \bar{\varphi}_{0}, \sin \bar{\psi}_{0} \sin \bar{\varphi}_{0}, \cos \bar{\psi}_{0}\right\}
$$

Here $\bar{\varphi}_{0}, \bar{\psi}_{0}$ are averages of the angles $\varphi_{0}, \quad \psi_{0}$ shown in Fig.2:

$$
F\left(\Delta_{\psi}, \Delta_{\varphi}\right)=\frac{1}{2 \pi \sigma_{\psi} \sigma_{\varphi}} \exp \left(-\frac{\Delta_{\psi}^{2}}{2 \sigma_{\psi}^{2}}\right) \exp \left(-\frac{\Delta_{\varphi}^{2}}{2 \sigma_{\varphi}^{2}}\right) .
$$

We assume, for simplicity, that $\Delta_{\psi}$ and $\Delta_{\varphi}$ are uncorrelated with $h_{0}$. In the figures starting from Fig.5, $\sigma_{\varphi}$ and $\sigma_{\psi}$ will be quoted in units of $\gamma^{-1}$. Then the resulting DR distribution, taking into account both, beam size and divergence, is given by the convolution of expression (9) with the distribution $F\left(\Delta_{\psi}, \Delta_{\varphi}\right)$ :

$$
\Lambda\left(K, \theta_{\psi}, \theta_{\varphi}\right)=\int \hat{I}\left(K, \theta_{\psi}-\Delta_{\psi}, \theta_{\varphi}+\Delta_{\varphi}\right) \cdot F\left(\Delta_{\psi}, \Delta_{\varphi}\right) d \Delta_{\psi} d \Delta_{\varphi}
$$

For the simulation it is convenient to substitute the angular deviations $\Delta_{\psi, \varphi}$ in Eq.(10) by the reduced ones $\delta_{\psi, \varphi}=\gamma \cdot \Delta_{\psi, \varphi}$, i.e. the electron angles measured in units of $\gamma^{-1}$, as it was the case for the photon angles $t_{\psi, \varphi}$. The numerical integration was performed under the simplified 
assumption of a triangular beam profile in the vertical direction (Fig.3b). After integration we can obtain the DR spectral-angular distribution which is possible to measure in an experiment. In Eq.(10) the integration over the variables $\Delta_{\psi}, \Delta_{\varphi}$ was extended over an interval $\pm 3 \sigma$ in each direction, and the integration step was chosen in such a way that the total error of simulation did not exceeded 3\%. As it was shown in Ref.[13], in its soft part (i.e. for $\omega \leq \omega_{c}$ ) the DR spectrum is determined mainly by the beam divergence, and on the contrary for DR photon energies $\omega>\omega_{c}$ by the beam size. Figs.5a-5b show simulation results for different values of the reduced photon energy $z$ (here and later we use the reduced variable $z=\frac{\omega}{\omega_{c}}=\frac{2 \pi a \sin \varphi_{0}}{\gamma \lambda}$ ). Fig.5 confirms that for small photon energies $(z \leq 1)$ the effect of the beam size is much smaller than the effect of the divergence. This result is consistent with the development of Eq.(8) at small $t_{\psi}, t_{\varphi}$ and $l$,

$$
\frac{d^{2} W}{d \omega d \Omega} \simeq \frac{\alpha \gamma^{2}}{\pi^{2}} e^{-z}\left[t_{\psi}^{2}+\left(1+\frac{z}{2}\right)^{2} t_{\varphi}^{2}+z^{2} l^{2}\right]
$$

In the following, we will assume that the beam is sufficiently narrow and well centered in the slit, so that $z^{2}<l^{2}>\ll 1, \gamma^{2}<\Delta_{\psi}^{2}>, \gamma^{2}<\Delta_{\varphi}^{2}>$ and neglect the beam size effect.

\section{Simulation results}

Fig. 6 shows the DR distributions from a slit in the direction perpendicular to the slit edge (i.e. for $\theta_{\psi}=0$ ), depending on the variable $\gamma \theta_{\varphi}=t_{\varphi}$, and Fig.7 in the plane $\theta_{\varphi}=0$ parallel to the edge, depending on the variable $\gamma \theta_{\psi}=t_{\psi}$ for different values $\sigma_{\psi}, \sigma_{\varphi}$. As it follows from that figures the distributions in the plane parallel to the edge (i.e. $t_{\psi}$ dependence) are more sensitive to a change in $\sigma_{\psi}$ or $\sigma_{\varphi}$ than in the perpendicular plane. More precisely, the values at origin are the same in Figs. 6 - 7 , since they correspond to $t_{\psi}=t_{\varphi}=0$ in both cases, but the maxima are lower for the $t_{\psi}$ distributions (because the exponential factor in Eq.(8) depends on $t_{\psi}$ ). As $\sigma_{\psi, \varphi}$ is increasing, the dependence in $t_{\psi}$ is considerably deformed, and it is possible to find a transition when the minimum at zero is changed into a maximum (see Fig.8). Thus, as a criterion we may take the ratio $R_{\|}=\frac{I_{\max }-I_{\min }}{I_{\max }+I_{\min }}[7]$, where $I_{\max (\min )}$ is the maximum (minimum) intensity of the DR spectral-angular distribution in the plane parallel to the slit edge. In the following we will consider only the parallel distribution and denote $R \equiv R_{\|}$. The value $\mathrm{R}$ depends on both $\sigma_{\psi}$ and $\sigma_{\varphi}$, as illustrated in Fig.9. The level lines $R=$ const, displayed in Figs.10-11 are well approximated by homothetic ellipses for not too large values of $\sigma_{\psi}$ and $\sigma_{\varphi}$. The ellipses were obtained by best fit adjustment of $a$ and $b$. Their semi-axes ratio $k=b / a$, more precisely, the mean of $k$ over 10 level lines of $R$ depends on $z$ as shown in Fig.12 and is well approximated by the linear function $k=1+\frac{z}{2}$ (this is also the result obtained by calculating $I_{\min }$ analytically using the approximation Eq.(11) and neglecting the dependence of $I_{\max }$ on $\sigma_{\psi}$ and $\sigma_{\varphi}$ ). For the case where $z=0$ we have transition radiation, characterized by azimuthal symmetry, and resulting in $\frac{b}{a}=1$. When the photon energy increases the ellipses become more and more elongated ( $k$ increases), i.e. the influence of the divergence $\sigma_{\psi}$ on the $t_{\psi}$ - dependence weakens in comparison to $\sigma_{\varphi}$. Fig. 13 shows $R$ as a function of

$$
b=\left[k^{2} \sigma_{\varphi}^{2}+\sigma_{\psi}^{2}\right]^{\frac{1}{2}}
$$


which is the semi-axis of the ellipse passing through the point $\left(\sigma_{\varphi}, \sigma_{\psi}\right)$. In addition, Gaussian approximations for the functional dependence $R(b)$ for different values of $z$,

$$
R \simeq \exp \left\{-\frac{b^{2}}{2 d_{b}^{2}}\right\}
$$

are depicted in Fig. 13. The width $d_{b}$ of these Gaussians is well described by a linear dependence on $z$, as illustrated in Fig.14.

As an application, the procedure is described how to derive the two unknown beam divergences from a simple experiment. Assuming a given DR photon energy, i.e., a fixed value of $z$, by Fig. 12 it is possible to obtain the semi-axes ratio $k=\frac{b}{a}$ of the ellipse characterizing the $\sigma_{\psi, \varphi}$ values of the initial beam, cf. Eq.(12). The remaining ellipse parameter $b$ is derived by the intensity ratio $R$ as measured in the experiment, using Eq.(13) and the value of $d_{b}$ extracted from Fig.14 with knowledge of $z$. By measuring $R$ for two fixed wavelengths $\lambda_{1}$ and $\lambda_{2}$ (i.e., two fixed relative photon energies $z_{1,2}=\frac{2 \pi a \sin \varphi_{0}}{\gamma \lambda_{1,2}}$ ) it is possible to define two ellipses of parameters $b_{1,2}$ and $k_{1,2}$ intersecting at the values of interest, $\left(\sigma_{\psi}, \sigma_{\varphi}\right)$ (see Fig.13). We have thus two equations for the determination of two unknown parameters $\sigma_{\psi}, \sigma_{\varphi}$ :

$$
k_{i}^{2} \sigma_{\varphi}^{2}+\sigma_{\psi}^{2}=b_{i}^{2}, \quad i=1,2
$$

\section{Summary and Conclusions}

In the present article formulas describing DR of a particle passing near the edge of a perfectly conducting half-plane screen are generalized to arbitrary angles $\varphi_{0}, \psi_{0}$ of the particle with respect to the target. It justifies the use, for any incidence angle, of previously given ultrarelativistic formulas, both with single-edge and slit targets. By means of these formulas, it is possible to take into account the beam divergence and to study its influence on the DR angular distribution from a slit in an exact way.

In a low emittance beam as for example in an electron storage ring the values for the horizontal and vertical divergences can have significantly different values. By the two-ellipse method described at the end of Section 5 it is possible to determine both parameters of the angular beam divergence, $\sigma_{\psi}$ and $\sigma_{\varphi}$, at least when the beam size effect can be neglected.

We have not estimated the precision of our method. This would require further simulations, including the beam size effect, the angular and spectral resolution and the sensitivity of the detector, etc. Here we only present the principle of the method. Two observed wavelengths should be as much different as possible to obtain ellipses of significantly different $b / a$ ratios, but $z$ cannot be chosen too large.

Our method can be complementary to the one proposed in [10]. They use two orientations of the slit as well as the information contained in the polarization of the radiation. We instead use the spectral information. Combining the information of the two methods should lead to a further improvement.

Acknowledgments. One of the authors (Potylitsina N.) wishes to thank the administration and the staff of the Institut de Physique Nucleaire de Lyon for their hospitality and the opportunity to perform this work, and to prof. Potylitsin A.P. for numerous discussions. 


\section{References}

[1] L.Wartski, S.Roland, J.Lassale, et al. Appl. Phys. -1975. -V.46. -P. 3644.

[2] X.Artru, M.Castellano, L,Catani, R.Chehab, et al. Nucl. Instr. And Meth.A 410 (1998) 148-158.

[3] D.W.Rule, R.B.Fiorito. Beam profiling with optical transition radiation. Proc. 1993 Particle Accelerator Conf., Washington, DC, USA. -P. 2453.

[4] A.P.Kazantsev, G.I.Surdutovich. Sov.Phys.Dokl.7 (1963) 990.

[5] D.M.Sedrakian. Izv. An ArmSSSR, 17(N4),1964, 103.

[6] M.L.Ter-Mikaelian. High Energy Electromagnetic Processes in Condensed Media, WileyInterscience, New York, 1972

[7] M.Castellano. Nucl. Instr. Meth. -1997. -V. A 394. -P. 275.

[8] A.P.Potylitsyn. Nucl. Instr. Meth. B -1998. -V.145. -P. 169-179.

[9] A.P.Potylitsyn and N.A.Potylitsyna. Russian Physics Journal, V-43, N-4, 2000; (arXiv, physics/0002034)

[10] R.Fiorito, D.Rule. Nucl. Instr. Meth. B-173, p.67, 2001

[11] B.M. Bolotovskii, E.A. Galst'yan. Uspekhi Fiz. Nauk 170 (2000) 8, Physics Uspekhi 43 (2000) 755

[12] R.B. Fiorito. Electron-Photon Interaction in Dense Media, ed. by H. Wiedemann, NATO Science Series II, vol. 49, p.91 (Kluwer Acad. Pub., 2002).

[13] J.Urakawa, A.Potylitsyn, T.Hirose et al. Nucl. Instr. Meth. -V. A472, -P. 309, 2001. 
a)

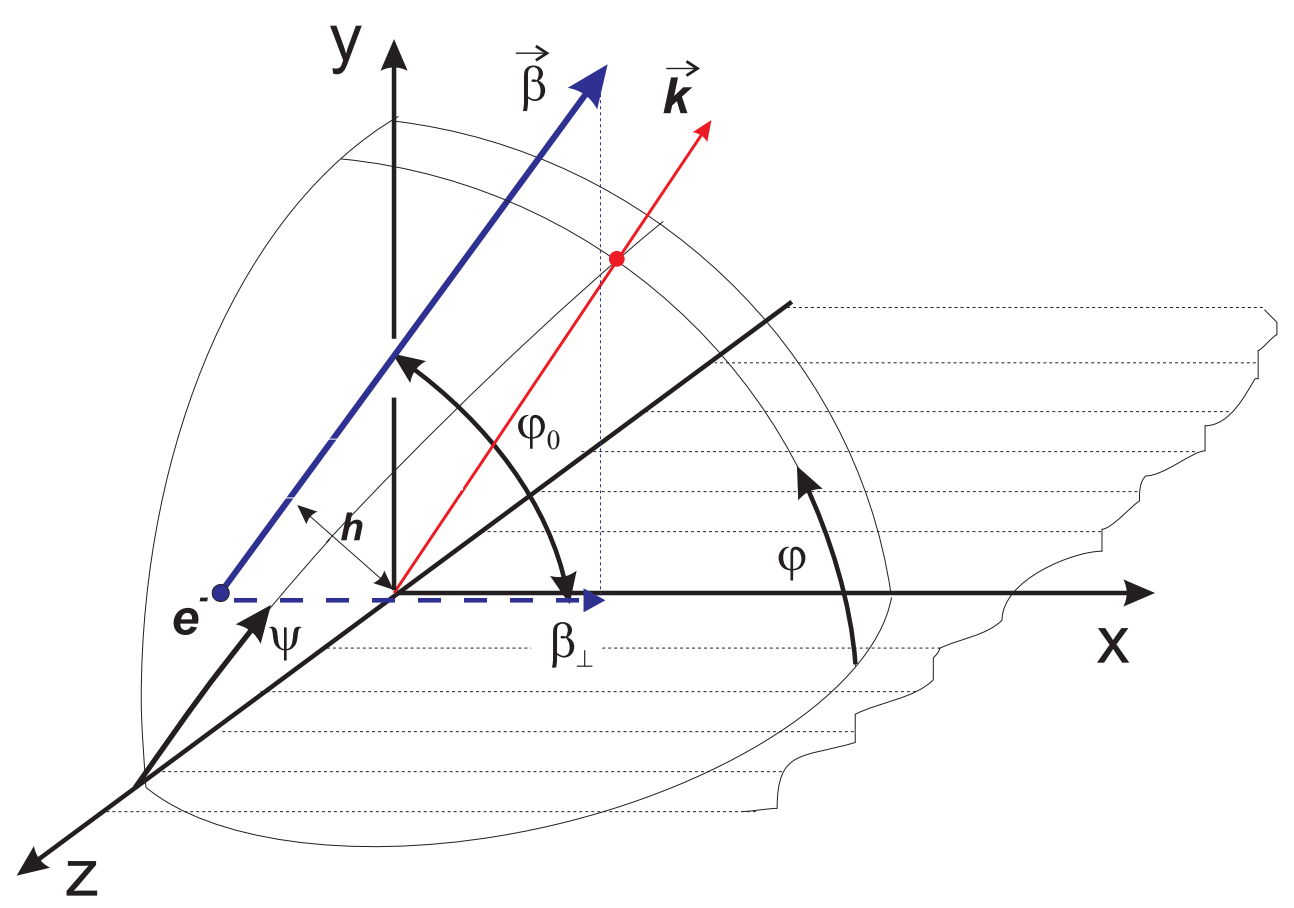

b)

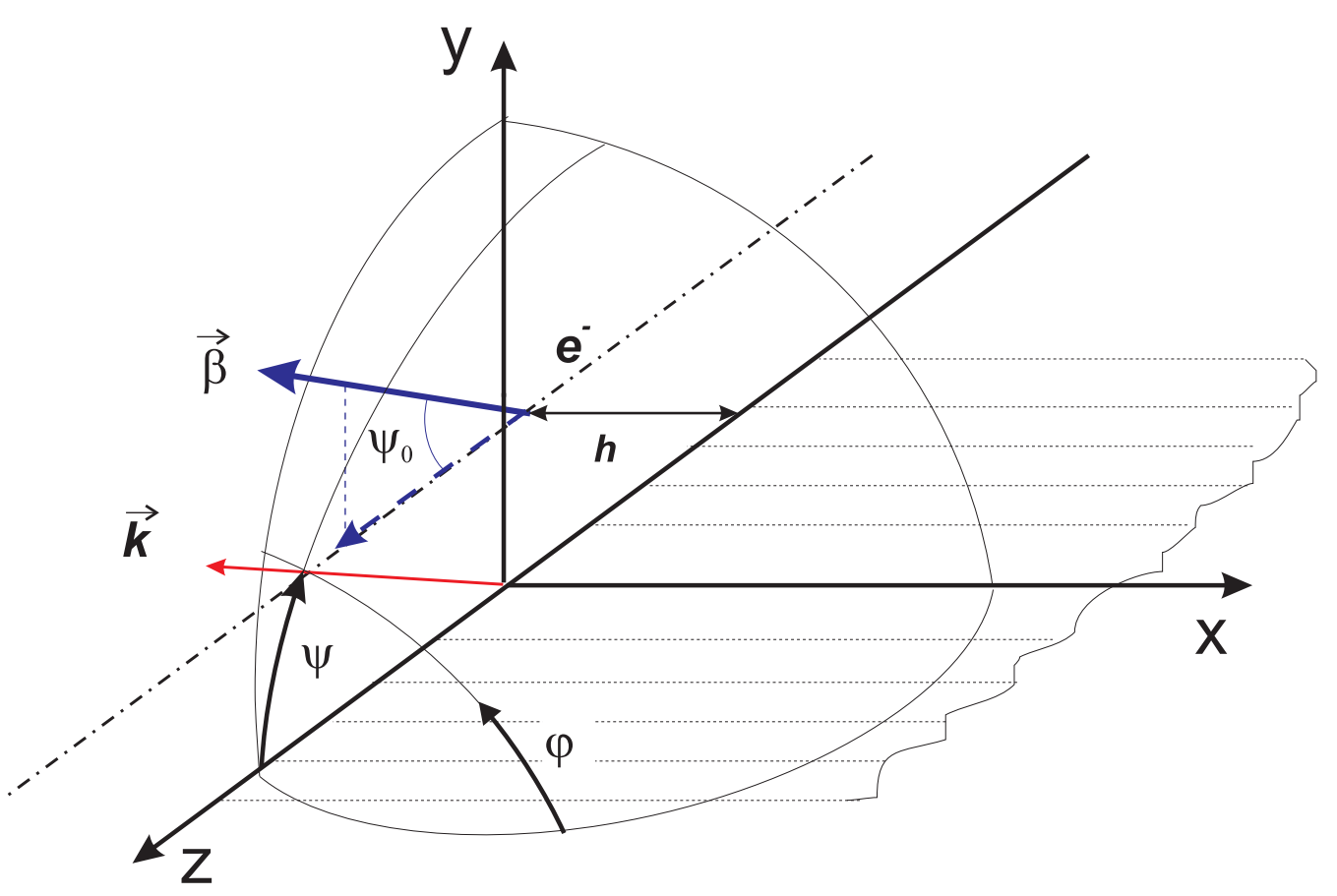

Fig. 1. Coordinate system for Diffraction Radiation produced by a charged partical moving in a) perpendicular geometry: the polar angle between electron beam and a target is $\psi_{0}=\frac{\pi}{2}$ b) parallel geometry: the azimuthal angle is $\varphi_{0}=\frac{\pi}{2}$. 
a)

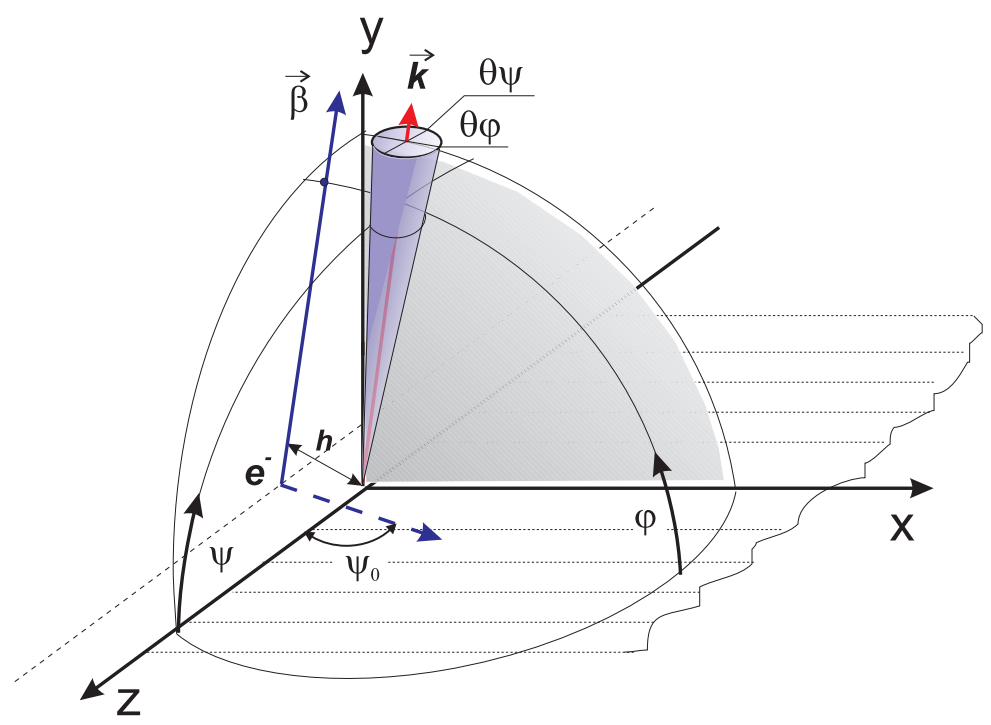

Fig. 2. Coordinate system for DR. General case, the electron beam has arbitrary angle $\varphi_{0}$, $\psi_{0}$ with respect to the target plane.

a)

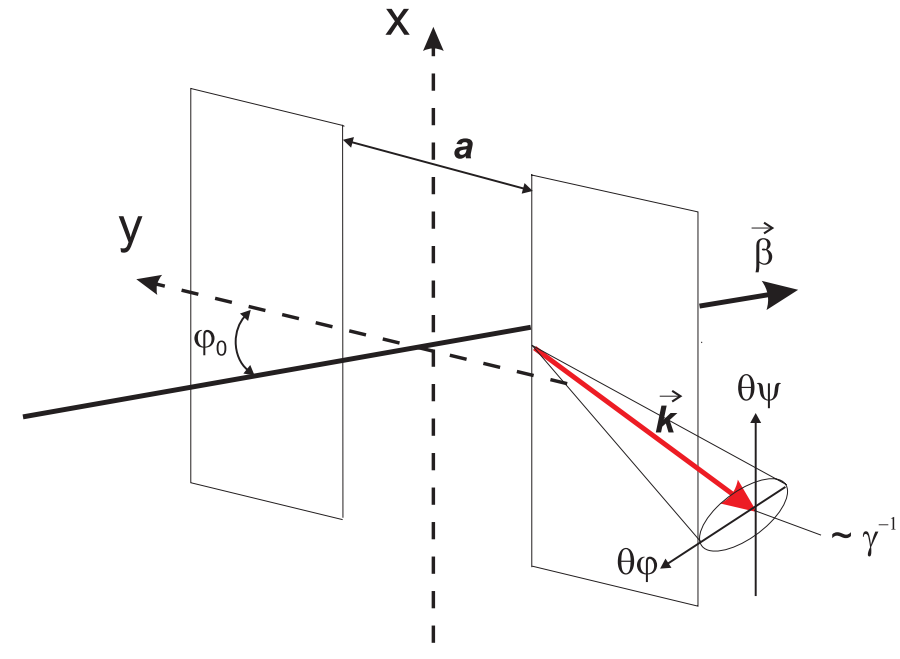

b)

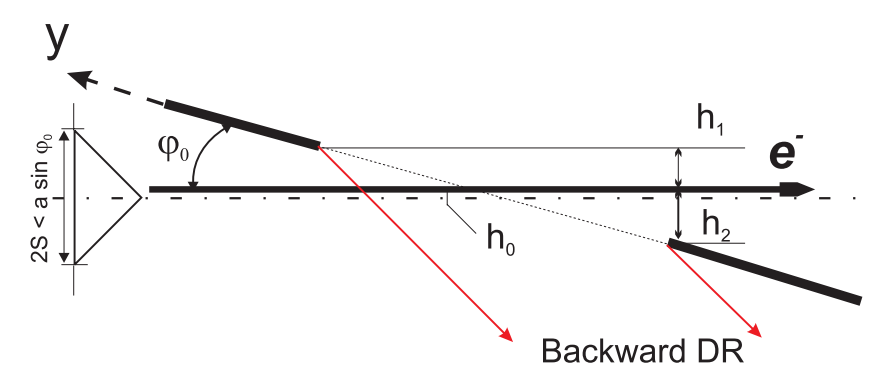

Fig. 3. a) General view of Backward Diffraction Radiation emitted by a particle passing through a slit, b) Top view. 


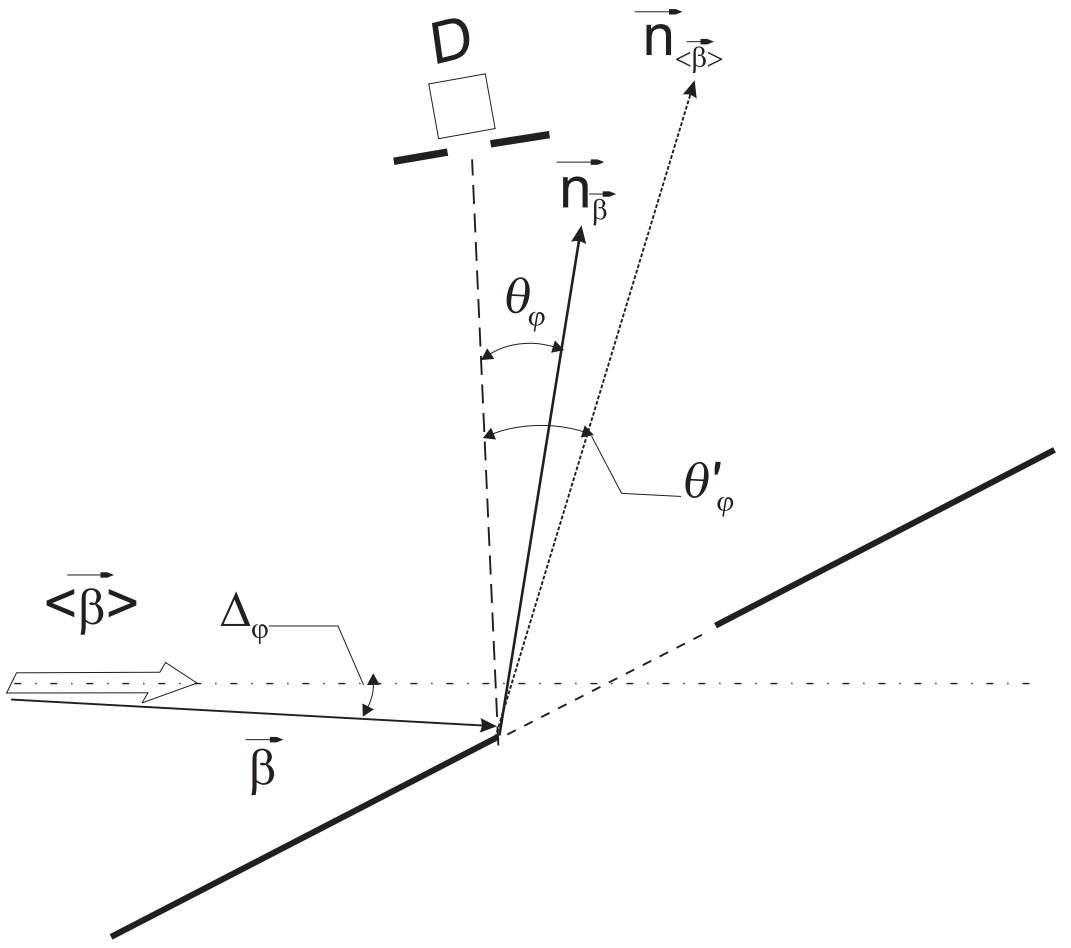

Fig. 4. Definition of the introduced angles.
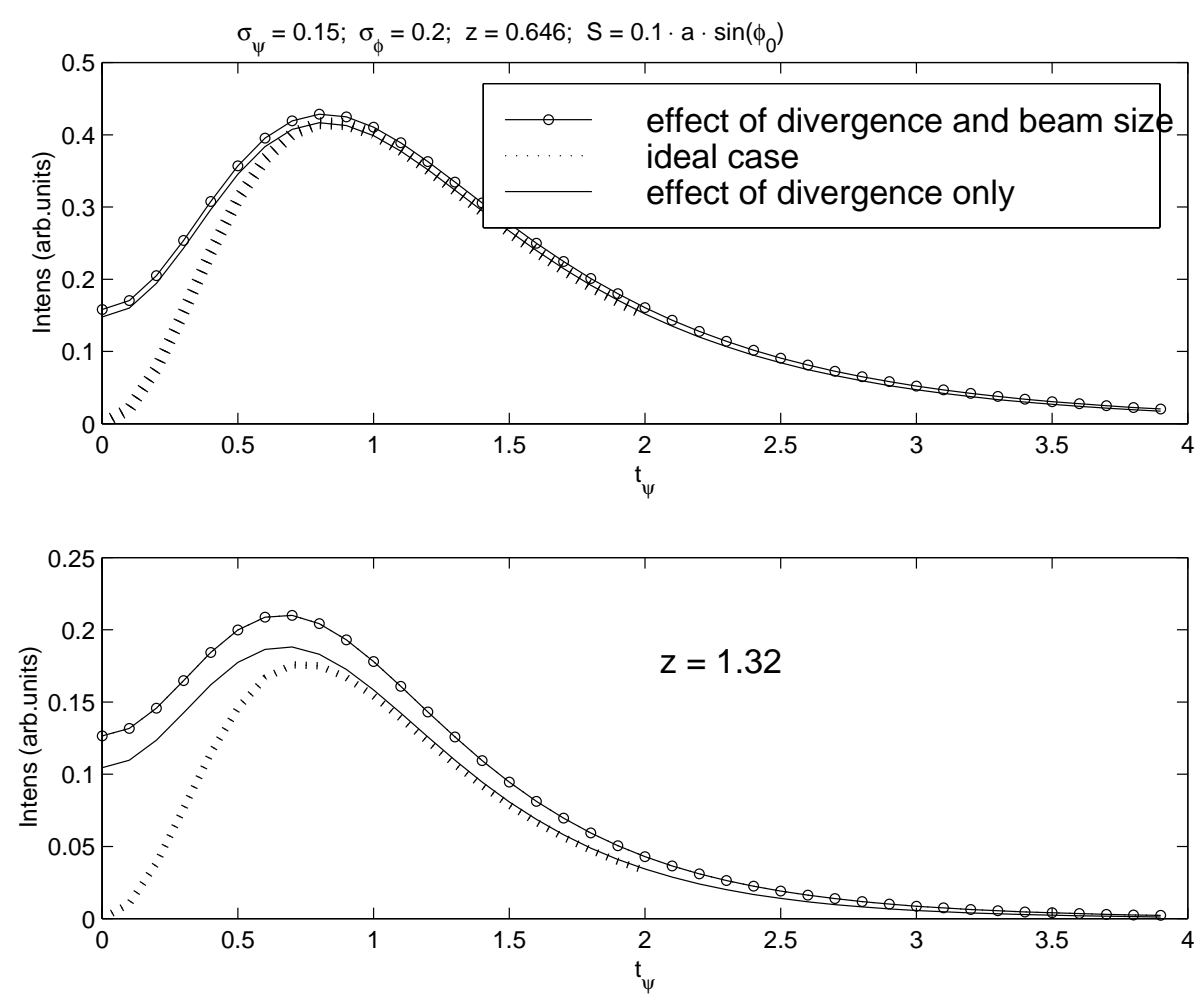

Fig. 5. Result of simulation of DR spectral-angular distribution for different values $z$. 

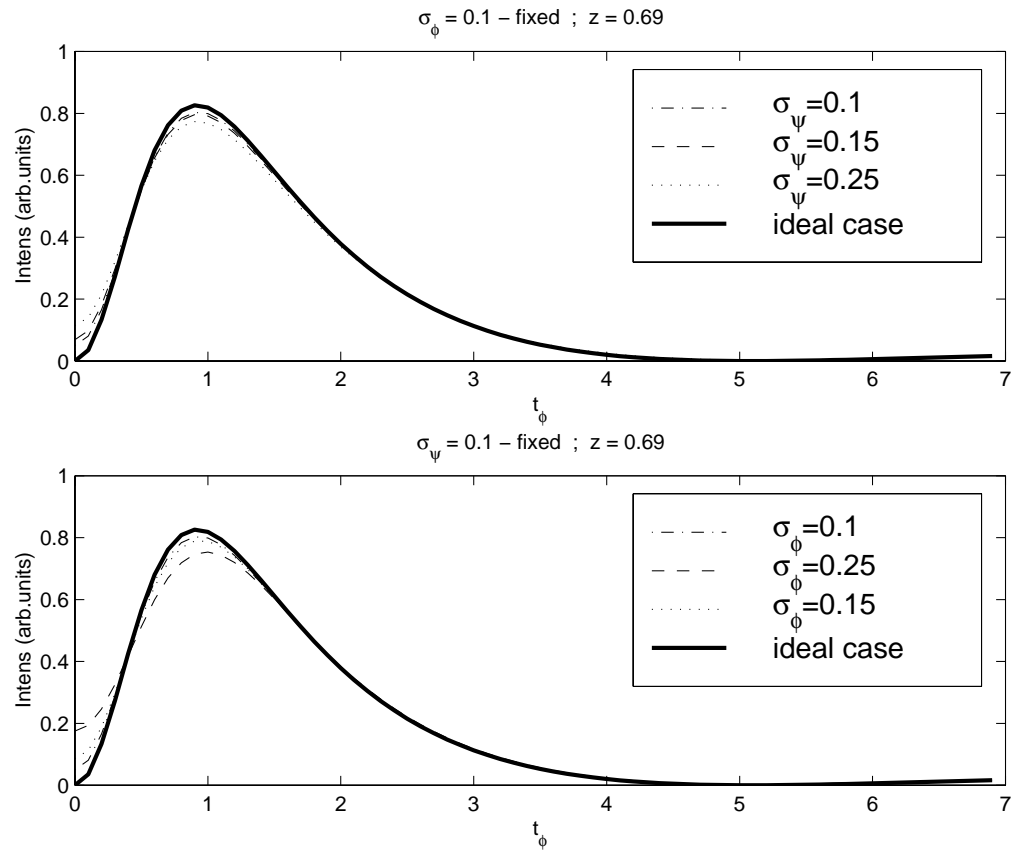

Fig. 6. Angular distribution in the horizontal plane, for different values of the horizontal and vertical divergences.
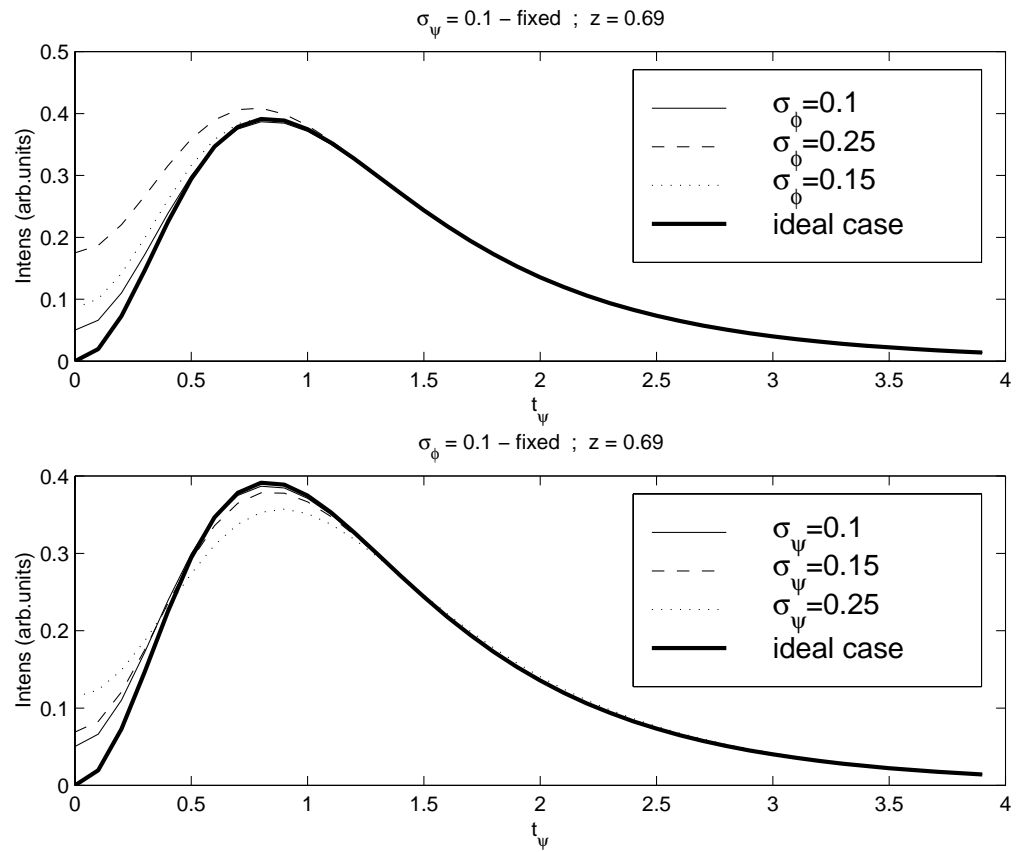

Fig. 7. Angular distribution in the vertical plane, for different values of the horizontal and vertical divergences. 


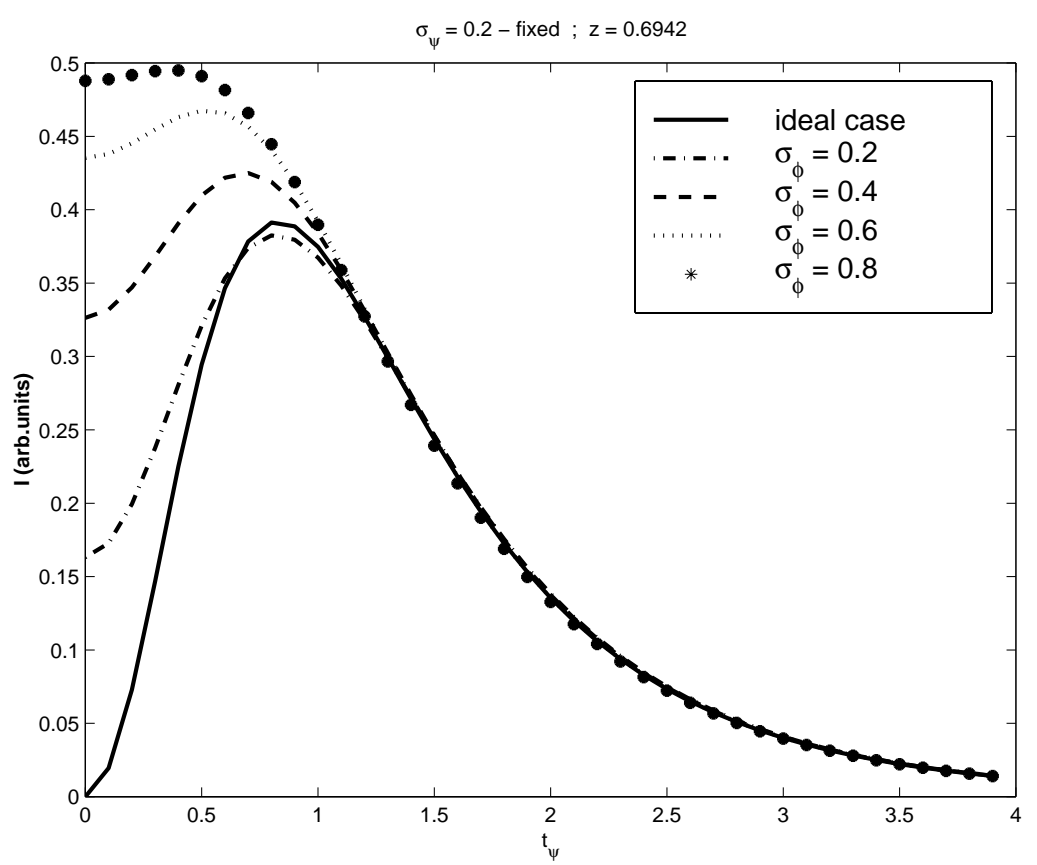

Fig. 8. Evolution of DR angular distributions with increasing of divergence in vertical plane.

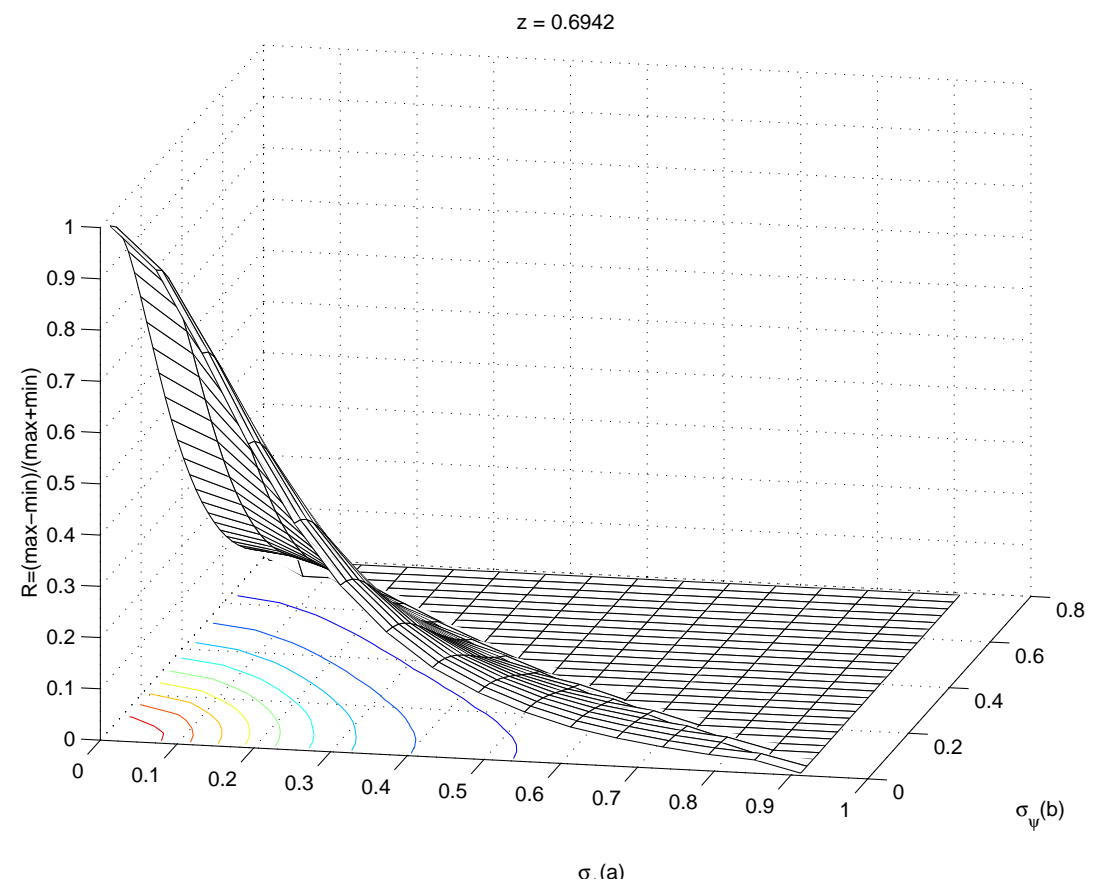

Fig. 9. Tree dimensional plot dependence of the parameter $\mathrm{R}$ on the beam divergence $\sigma_{\psi}, \sigma_{\varphi}$ 


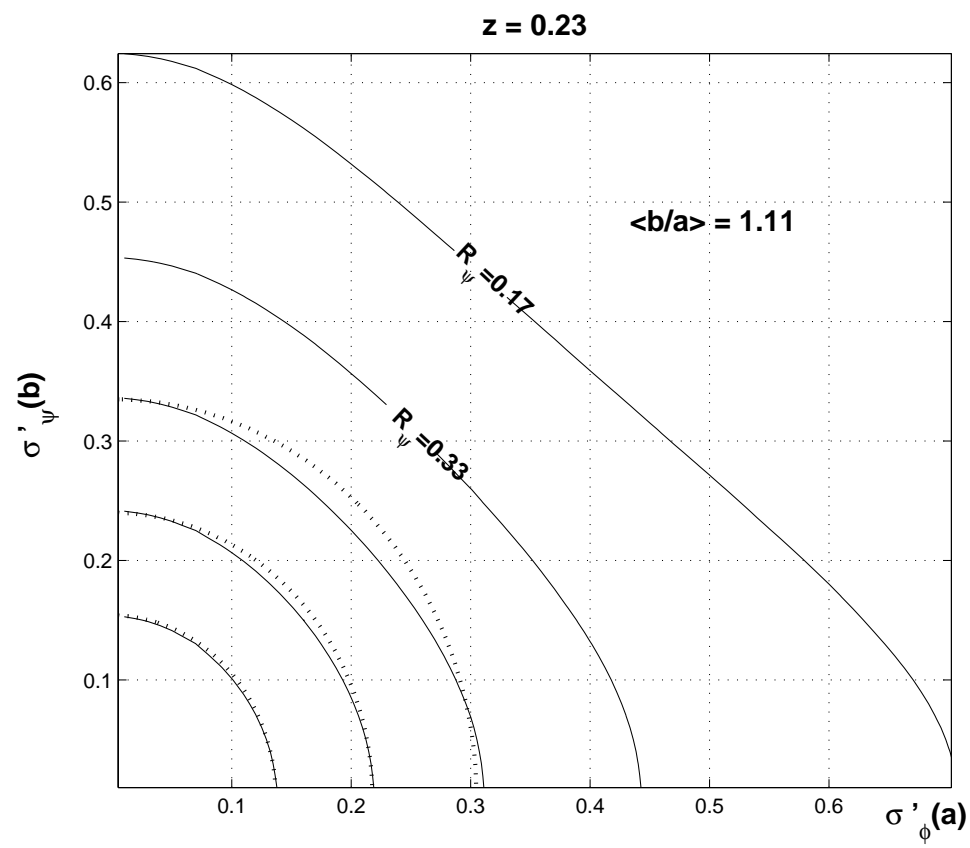

Fig. 10. Level lines for parameter $R\left(\sigma_{\psi}, \sigma_{\varphi}\right)=$ const for $z=0.23$ - solid line and dotted line for ellipse approximation.

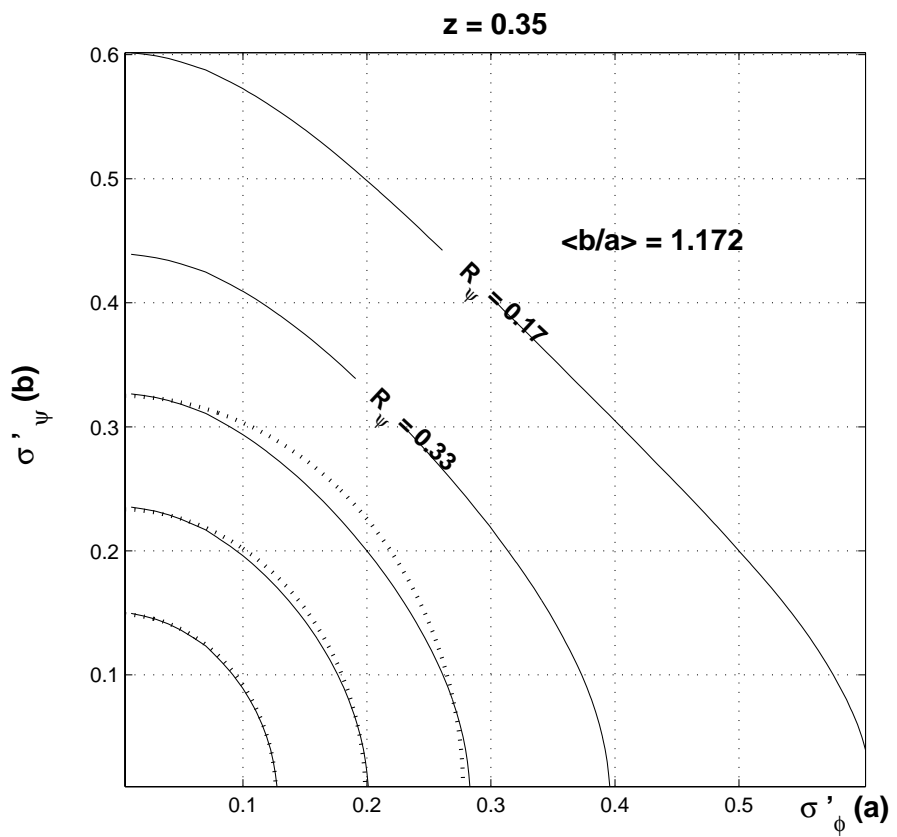

Fig. 11. Level lines for parameter $R\left(\sigma_{\psi}, \sigma_{\varphi}\right)=$ const for $z=0.35$ - solid line and dotted line for ellipse approximation. 


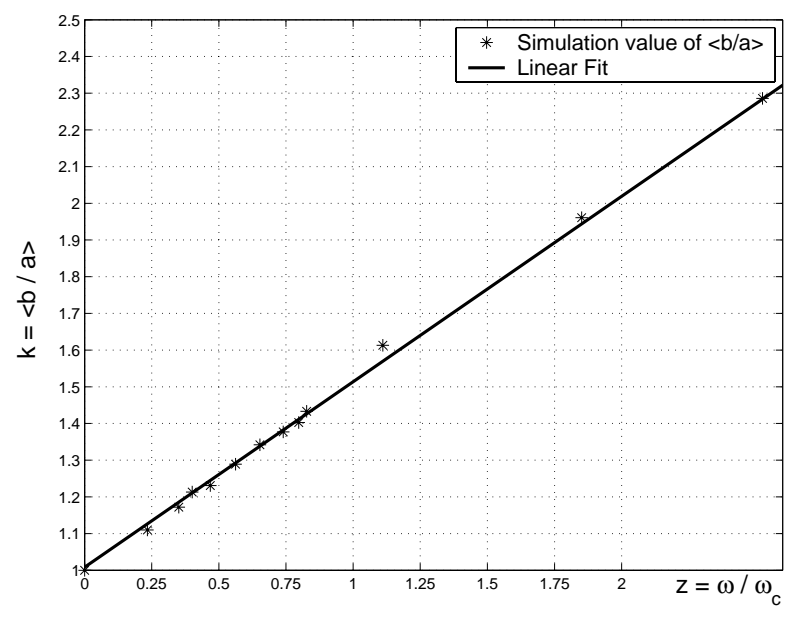

Fig. 12. Dependence of the mean ratio $\frac{b}{a}$ on DR wavelength $z=\frac{\omega}{\omega_{c}}$.
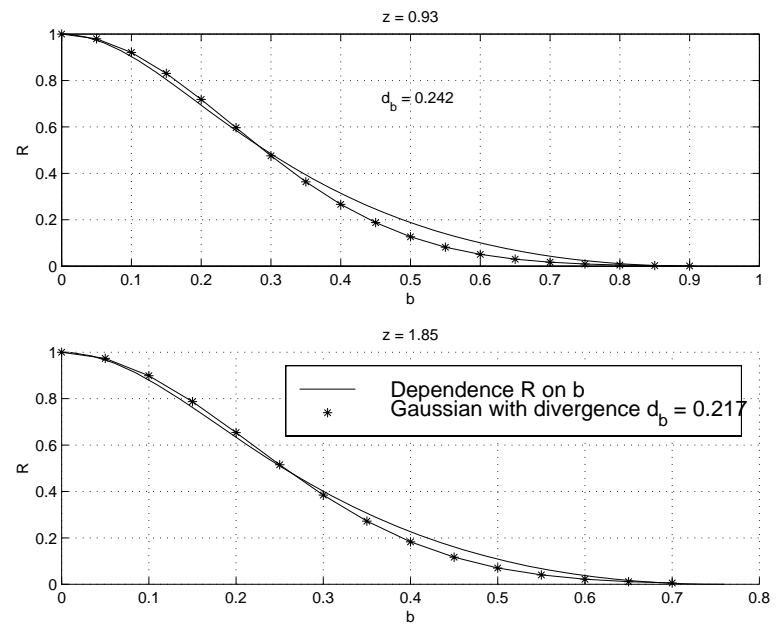

Fig. 13. Functional dependence of the intensity Ratio $R$ on the ellipse axis $b$ for different values of the normalized frequency $\mathrm{z}$ (solid lines). In addition the Gaussian approximation for $\mathrm{R}$ with the optimized standard deviation $d_{b}$ is drawn (stars).

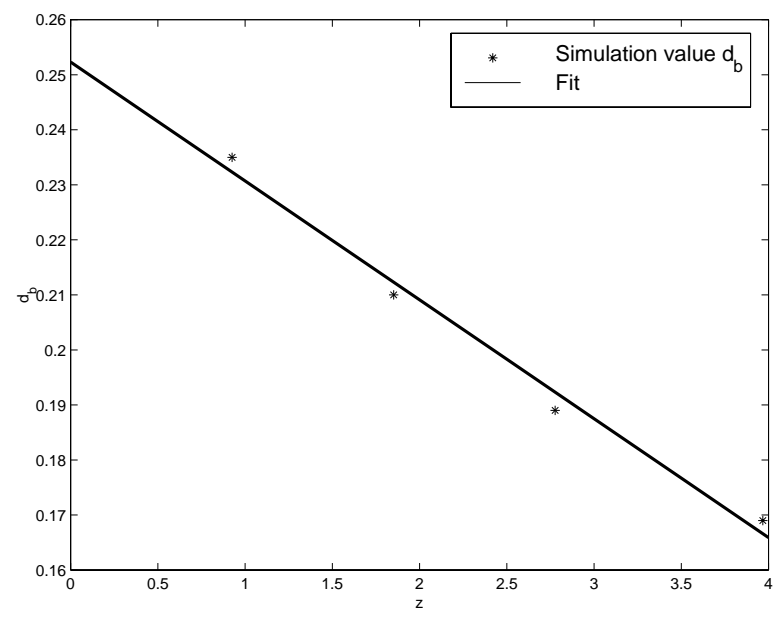

Fig. 14. Dependence of the optimized standard deviation $d_{b}$ in Gaussian approximation for $\mathrm{R}$ on the normalized frequency $z$. 